\title{
Factores Psicológicos Asociados a la Maternidad Adolescente en Menores de 15 años. ${ }^{1}$
}

\section{Psychological Factors Associated with Adolescent Motherhood in Mothers Under 15 Years Old}

\author{
Maruzzella Valdivia P.* \\ Marta Molina S.
}

\begin{abstract}
Resumen
La presente investigación analiza variables psicológicas asociadas a la maternidad de mujeres adolescentes menores de 15 años, comparándolas con madres entre 20 y 34 años y adolescentes no madres ni embarazadas, en un estudio de tipo transversal descriptivo comparativo. Las madres adultas no presentan niveles de apoyo social percibido significativamente mayores que las madres adolescentes menores de 15 años ( $\mathrm{p}=0.46)$, así como tampoco un nivel intelectual significativamente mayor que las madres adolescentes menores de 15 años $(\mathrm{p}=0.23)$. Además, no se observó un nivel de autoestima total significativamente mayor que las madres adolescentes menores de 15 años $(p=0.5)$. No obstante, las adolescentes madres evidencian significativamente mayor nivel de autoestima social que las madres adultas $(p=0,036)$.Las adolescentes no madres no presentaban un nivel de apoyo social significativamente mayor que las adolescentes madres $(p=0.49)$. Sin embargo, en la subescala amigos las adolescentes no madres presentaban significativamente mayor nivel de apoyo social percibido que las adolescentes madres $(p=0,045)$ y en la subescala apoyo social percibido otros, las embarazadas adolescentes mostraron significativamente más apoyo social que las adolescentes no embarazadas $(\mathrm{p}=0,002)$. Por otra parte, las adolescentes no madres exhibieron un nivel intelectual significativamente mayor que las adolescentes madres $(\mathrm{p}=0.017)$. Las adolescentes madres no evidenciaron un nivel de sintomatología depresiva significativamente mayor que las adolescentes no madres. Por el contrario estas últimas fueron las que mostraron, significativamente, un mayor nivel de sintomatología depresiva $(p=0.032)$. Las adolescentes no madres no presentaron un nivel de autoestima significativamente mayor que las adolescentes madres $(\mathrm{p}=0.12)$. Sin embargo, se observó diferencias débilmente significativas
\end{abstract}

1 Este trabajo es parte de una iniciativa más amplia que corresponde al proyecto de investigación, de la Dirección de Investigación de la Universidad de Concepción N²000-84B009-1-0, titulado "Embarazo en Adolescentes Menores de 15 años: Una Evaluación desde la Interdisciplina", coordinado por la profesora Marta Molina, docente de la Universidad de Concepción. Parte de este trabajo, además, corresponde a la Tesis para optar al Grado de Magíster en Psicología de la Salud de la autora principal, docente de la Universidad de Concepción.

* Psicóloga, Magíster en Psicología de la Salud. Académica, Universidad de Concepción.mavaldivia@udec.cl 
en las subescalas escolar $(p=0,07)$ y general $(p=0,099)$ a favor del grupo de madres adolescentes en relación al grupo de adolescentes no madres. Los resultados obtenidos invitan a reflexionar acerca del rol del desarrollo cognitivo, en particular el coeficiente intelectual y la fábula personal. La conjugación de estos factores deja a las menores y a su hijos/as en una situación de riesgo y vulnerabilidad, necesaria de atender en futuras investigaciones e intervenciones.

Palabras Clave: Embarazo Adolescente - Menores de 15 años - Variables Psicológicas.

\begin{abstract}
The present research analyzes psychological variables associated with Adolescent motherhood; comparing young mothers under 15 years old, with mothers between 20 and 34 years old, and adolescents non mothers neither pregnant in a comparative descriptive transversal study. The adult mothers didn't show significant higher levels of perceived social support than adolescent mothers under 15 years old $(p=0.46)$, neither a significant higher intelectual level than adolescent mothers under 15 years old $(p=0.23)$. Besides, it didn't show a higher total self-steem level than adolescent mothers under 15 years old $(p=0.5)$. Nevertheless, the adolescent mothers had a significant higher level of social self-steem than the adult mothers $(\mathrm{p}=0.036)$. The adolescents non mothers, didn't show a higher level of perceived social support, than the adolescents mothers $(p=0.45)$ and in the sub-scale, perceived social support from others; the adolescent mothers had higher levels of perceived social support than the non pregnant adolescents $(p=0.002)$. On the other hand, the adolescents no mothers showed a significant higher intelectual level than the adolescens mothers $(p=0.017)$. The adolescent mothers didn't show a higher level of depressive symptomatology than the adolescents non mothers. On the contrary, the adolescent non mothers showed significant higher levels of depressive symptomatology $(\mathrm{p}=0.032)$. The adolescent non mothers didn't had a significant higher level of self-steem than the adolescent mothers $(p=0.12)$. However, a weak significant differences was found in school self-steem $(p=0.07)$ and in general self-steem $(p=0.09)$, the adolescents mothers having higher average than the adolescent non mothers. The findings invite us to think about cognitive development, particulary on the IQ and the personal narrative. The interaction of both factors leave the adolescents and their sons and daughters in a risky situation and vulnerability, important to consider it in future research and interventions
\end{abstract}

Key Words: Adolescent Pregnancy - Under 15 years old - Psychological Variables.

\section{Introducción}

Si bien el embarazo adolescente ha sido estudiado desde una perspectiva biopsicosocial desde hace décadas, llama la atención la escasa literatura existente en torno al segmento etáreo correspondiente a las menores de 15 años; ya que si bien en términos generales, la adolescencia es una etapa compleja del desarrollo, donde se consolidan gran parte de las características personales, y además, donde existe una cierta vulnerabilidad para la aparición de trastornos psicológicos y problemas psicosociales. (Vives y col., 1992), el período comprendido entre los 10 y los 14, denominado adolescencia inicial (Florenzano, 1997) presenta características específicas de consolidación de las estructuras cognitivas y 
afectivas que lo vuelven clave para el desarrollo de una vida adulta normal.

En este período, son incipientes aún las estrategias de autodefinición que la niña o el niño toman para conformar la propia identidad, lo que los vuelve aún más vulnerables a un desarrollo alterado.

Asociado al cambio físico emergente en esta etapa viene un despertar sexual, que implica un creciente e intenso impulso e interés en esta área (Jaskiewicz \& McAnarney, 1994; Montenegro \& Guajardo, 1994).

A pesar de que este tránsito de acercamiento a la sexualidad es gradual y parece ser más lento en las mujeres que en los hombres (Hoffman et al., 1997), existe un porcentaje de menores que adelantan este proceso iniciándose sexualmente antes de los 15 años de edad, como se observó en esta investigación.

En el área cognitiva se inicia el avance desde un razonamiento concreto a un pensamiento lógico abstracto, el que se caracteriza por ser hipotético deductivo, integrando mejor lo que se ha aprendido en el pasado con los problemas presentes y su planeación en el futuro, además permitiendo prevenir posibles consecuencias antes que éstas sucedan o en situaciones que no han ocurrido nunca, ya que no se limita a la experiencia (Hoffman et al., 1996; Papalia\& Wendkos, 1998).

No obstante, en una investigación realizada en Chile por Alamo et al. (1981), se mostró que a la edad de dieciséis y diecisiete años, aún existía un predominio del pensamiento concreto (citado en Florenzano, 1997). Esta relatividad en el avance en las etapas del desarrollo cognoscitivo es coherente con algunas de las líneas de critica al pensamiento Piagetano. Una de las más importantes viene desde autores como Vigotsky, Ausubel y muchos otros, quienes señalan que la socialización y la instrucción juegan un rol fundamental en el desarrollo del pensamiento (Pozo, 1997). Esto implica que es altamente probable que las jóvenes de 15 años o menores, especialmente de niveles socioeconómicos deprivados y/o con un menor nivel instruccional, no hayan desarrollado a cabalidad (o tal vez nunca lo hagan) un tipo de pensamiento que les ayude a planificar el futuro de una manera más realista y flexible; integrando sus experiencias previas y sabiendo cuales serán las consecuencias de sus acciones.

Otra particularidad del razonamiento, sería el egocentrismo, el que se caracterizaría en esta etapa según Elkind (1985), por la creencia de una audiencia imaginaria, es decir el pensar que las otras personas están constantemente preocupadas de ellos, lo que los hace muy conscientes de si mismos; y la fábula personal que se refiere a un sentimiento típico del adolescente de ser especial y úni$\mathrm{co}, \mathrm{y}$ no estar sujeto a las normas que rigen el resto del mundo. Estas formas de pensamiento parecen predominar según lo que plantea Elkind en la primera fase de la adolescencia (citado en Hoffman et al., 1996).

Si consideramos la fábula personal y añadimos la conducta exploratoria propia de la adolescencia, que está relacionada con la búsqueda de la identidad como se verá más adelante, no es extraño que los adolescentes se expongan a riesgos que a juicio de los adultos son innecesarios (Rice, 1997). Asociando esto último con el tema del embarazo adolescente, se entendería cómo estas menores se exponen a tener relaciones sexuales desprotegidamente. Siguiendo en esta línea, se encuentran los hallazgos de Corona y Tidwell (1999), que observaron que las adolescentes madres, pensaban que criar a un hijo iba a ser mucho más fácil y entretenido de lo que realmente es.

Si cruzamos esta información con aquella relacionada con la prevalencia y magnitud del problema, llegaremos a conclusiones preocupantes. En nuestro país, según el INE, en casi dos décadas, la tasa de fecundidad en muje- 
res entre 15 y 19 años ha aumentado levemente de 69.7 a 70.2 nacidos vivos por mil adolescentes. En Chile, nacen cada año alrededor de 40.300 niños cuyas madres tienen entre 15 y 19 años. A esta cifra se agregan otros 1.175 nacimientos en menores de 15 años. De esta forma, en nuestro país la incidencia de embarazo en la adolescencia es alrededor de $16.16 \%$, cifra muy similar a la de la octava región de $16.20 \%$ (INE, 2000). Específicamente, en el segmento de menores de 15 años, se observa el incremento en países como México (Lartigue et al., 1992) y Estados Unidos (Jaskiewicz \& McAnarney, 1994).

En Chile, las cifras muestran un aumento en el embarazo de niñas menores de 17 años. Así, a pesar de que la tasa de fecundidad global en nuestro país, para las mujeres de 20 años y menos ha disminuido entre 1980 y 1998, para el grupo de mujeres menores de 15 años la tasa específica de fecundidad por mil ha aumentado de un 1.6 en 1980 a un 2.2 en 1998. En la octava región esta tasa es mayor que la tasa nacional, alcanzando 2.4 puntos (INE, 2000).

A pesar de la complejidad descrita, son más bien pocas las investigaciones centradas en el embarazo adolescente en esta época. Probablemente esta escasez se debe más que nada a la dificultad para acceder a muestras correspondientes a este rango de edad.

La presente investigación, y el proyecto más amplio en el cuál se inserta, busca responder a esta necesidad, y profundizar el conocimiento existente al respecto.

\section{Consecuencias psicosociales ${ }^{2}$}

La adolescente madre debe asumir una multiplicidad de roles, que son aquellos que conlleva una tarea de adultos como es la ma- ternidad, para los cuales no está psicológicamente madura, como se explicó en el apartado anterior, ya que, sigue siendo niña cognitiva, afectiva y económicamente (Muñoz et al., 2001; Restrepo, 1991; Vera, Gallegos \& Varela, 1999), agudizándose esto aún más en el caso de la primera adolescencia.

Si lo habitual es que todo el grupo familiar se debe adaptar para recibir a un nuevo miembro (Hernández, Kimelman \& Montino, 2000), esto es mucho más evidente en la adolescencia (Vives et al., 1992), ya que las familias de las adolescentes suelen ser más complejas, ya que incluyen a sujetos en diversas etapas del desarrollo; los que probablemente no están esperando la llegada de un nuevo integrante, que de alguna manera va a modificar sus vidas.

En Chile, el 95\% de los hijos de mujeres menores de 15 años nacen fuera del matrimonio, mientras que en la población general el porcentaje alcanza el 45\% (INE, 2000). A esto se agrega que entre los años $1980 \mathrm{y}$ 1998, el porcentaje de hijos nacidos fuera del vínculo matrimonial en las menores de 15 ha aumentado de $66,6 \%$ a $95.1 \%$ (INE, 2000).

\section{Consecuencias culturales-económicas}

Otra consecuencia evidente de esta situación es la deserción escolar. Así, en una investigación realizada en E.E.U.U., se observó que alrededor del 50\% de las embarazadas adolescentes abandonaron la educación formal (Carver, Kittleson \& Lacey, 1990) Esto también ha sido encontrado en Chile en una serie de estudios; los que muestran un porcentaje cercano al $70 \%$ de deserción escolar entre adolescentes embarazadas (Burrows, Rosales \& Muzzo, 1994; Ferrada et al., 2002; Muñoz et al., 2001; Rojas, 1991; Vera, Gallegos \& Varela, 1999).

2 Para los fines de la presente investigación no se expondrán aquellos aspectos relacionados con el ámbito bíomédico.

Estos están descritos en la Tesis en la cual este artículo está basado. 
Por otra parte, las adolescentes no madres presentan mayores perspectivas educacionales y planes futuros, en relación a las embarazadas (Guijarro et al., 1999) lo que trae aparejado menores logros educacionales y laborales (Florenzano,1997).

Este fenómeno ayuda a explicar los mecanismos de la transmisión intergeneracional de la pobreza (Brandell y Cifuentes, 2000; Corcoran, 1999; Muñoz et al., 2001), ya que tendríamos a una niña pobre que tiene un hijo a temprana edad, y que asociado a ésto, deserta de sus estudios, o que en algunos casos, ya los había dejado previamente. Por lo tanto, podrá aspirar sólo a trabajos mal remunerados debido a su baja calificación, si es que puede trabajar, es decir si cuenta con quien dejar a su hijo.

Otros efectos señalados, en la literatura, con relación a los hijos e hijas de las adolescentes son el maltrato infantil y la morbilidad infantil (Muñoz et al., 2001; Restrepo, 1991) ${ }^{3}$, así como el menor desarrollo cognitivo de los menores. Esto último se observó en Estados Unidos, en un estudio longitudinal llevado a cabo con 70 madres adolescentes con sus hijos, demostrando que estos últimos quedaban en el rango límite del desarrollo del lenguaje. En términos intelectuales, el 56\% de los niños tenían un CI bajo lo normal y un 23\% mostraban problemas conductuales (Miller, Miceli, Whitman \& Borkowsky, 1996).

\section{Factores de riesgo}

Kalil y Kunz (1999), señalan que el efecto acumulativo de los factores de riesgo es particularmente significativo en el embarazo adolescente. A partir de una muestra de 958 adolescentes, en todo Estados Unidos, se llegó a la conclusión que si éstas presentaban 5 o más factores de riesgo sociodemográficos, tenían 16 veces más posibilidades de quedar embarazadas durante la adolescencia que sus contrapartes con solo un factor de riesgo.

Con relación a los factores predisponentes, se mencionan en la literatura elementos biológicos, psicosociales y psicológicos. Dentro de los factores biológicos llama la atención la menarquia más temprana y el hecho de que haya sido madre previamente. (Molina et al.,1997, Issler, 2001; Davis, 1989, cit. en Castro y Matamala, 1996). Dentro de los factores psicológicos y sociales, la discusión ha sido un poco más extensa (Muñoz, 1993).

\section{Factores sociales}

En el ámbito sociocultural se ha indicado que entre un $33 \%$ y un $46 \%$ de las adolescentes embarazadas, han abandonado la escuela antes de quedar encinta ( Maynard, 1995, cit, en Levine \& Chase-Landsdale, 1998). En Brasil, se encontró que un $84,5 \%$ de adolescentes gestantes ya se encontraban fuera del sistema educacional, al embarazarse (Fujimori et al., 1997). Estas jóvenes presentan menores niveles de escolaridad que embarazadas adolescentes que han desertado durante su estado de gravidez y a menudo una profunda deprivación socioeconómica (Ferrada et al., 2002).

En la Octava Región, Chile, según resultados obtenidos por Molina et al. (1992) y Ferrada et al. (2002), alrededor de un $40 \%$ de adolescentes gestantes, ya había desertado del sistema escolar y se encontraba sin realizar ningún tipo de actividad productiva, previo al embarazo.

Por otra parte, Evans y Rosen (2000), han comprobado que al mantenerse las mujeres en el sistema de educación formal, ellas tienden a posponer su primer embarazo, a usar métodos anticonceptivos, a tener un menor número de hijos y a responder mejor al cuidado de ellos.

3 Para una actualizada revisión, en cuanto a intervenciones en esta línea, se sugiere a Traid (1999). 
Se ha reportado además que ellas presentan un desempeño escolar pobre (Short \& Slusher, 1994; Guijarro et al, 1999), con frecuentes repitencias de curso (Ferrada et al., 2002), o con un desinterés por un futuro ligado a los estudios (Arriagada et al., 1991; Florenzano, 1997; Issler, 2001)

Se ha indicado que las mujeres que llegan a ser madres durante la adolescencia tienen menores niveles educacionales que quienes postergan el embarazo hasta la adultez (Molina et al., 1997; Stevens-Simon \& Lowy, 1995; Vera et al., 1999).

En relación al nivel socioeconómico, diversas investigaciones tanto nacionales como internacionales coinciden en señalar que las jóvenes que se embarazan precozmente, generalmente pertenecen al nivel socio económico bajo, entre las que se pueden mencionar en E.E.U.U a Corcoran, Franklin y Bennet (2000), Desmond (1994), y Lagana (1999). En Latinoamérica; Guijarro et al. (1999) en Ecuador, y Rojas (1991) en Costa Rica, en Brasil, Fujimori et al. (1997). En Chile, con resultados equivalentes, cabe mencionar a Arriagada et al. (1991), Molina, Peña y Quiroz (1992), Salinas, Pérez, Ramírez y Cumsille, (1997).

La falta de oportunidades para el futuro, asimismo, ha sido mencionada como un factor predisponente para este fenómeno (Desmond, 1994). ${ }^{4}$

\section{Factores Psicológicos}

En este ámbito se considera de relevancia analizar las conductas de las menores que se asocian al embarazo, las características de sus familias, el apoyo social percibido, el nivel intelectual de estas jóvenes, su autoestima y la presencia o ausencia de sintomatología depresiva; todas variables que han aparecido como importantes en la literatura relacionada con esta problemática.

\section{Conductas de riesgo}

Conductualmente se ha indicado como frecuente en este grupo de riesgo el consumo de alcohol, el uso de drogas, una actividad sexual precoz, o la existencia previa de un aborto espontáneo (Coard, Nitz \& Felice, 2000; Horwitz, Klerman, Kuo, \& Jekel, 1991ª Medora \& Von der Hellen, 1997; Paikoff, 1995; Peterson \& Crokett, 1992; Short \& Slusher, 1994; Urdy, Kovenock, \& Morris, 1996, cit. en Levine y Chase-Landsdale, 1998;). En México, Gutiérrez, Pascacio, De la Cruz y Carrasco (2002), han reportado lo mismo.

Además se ha señalado que hay un bajo índice de uso de medidas anticonceptivas en la primera relación sexual, y que esto es más frecuente en las adolescentes de menor edad (Abma et al., 1997, cit. en Levine y ChaseLandsdale, 1998; Fujimori et al., 1997; Gutierrez, et al., 2002; Lagana, 1999; Molina et al., 1997; Vera et al., 1999). De hecho, en un estudio realizado en la ciudad de Santiago de Chile con embarazadas adolescentes, se encontró que la joven se expone un año o más al coito sin protección, antes de solicitar contracepción. (Varas, Kramarosky, Díaz, Sibilla, \& Gaete, 1999).

Siguiendo esta línea de investigación los trabajos realizados en la ciudad de Concepción por Ferrada et al. (2001) y Molina et al. (2001) muestran porcentajes de utilización de anticonceptivos del orden del 5,8\% y del $18,1 \%$, respectivamente, en adolescentes de 15 años o menos, previo a su embarazo. Ambos porcentajes son significativamente menores a los encontrados en los grupos control de adultas embarazadas (20-34 años).

\footnotetext{
4 Se debe señalar, sin embargo, que más allá de lo evidente que pudiera ser para el lector el impacto de la deserción escolar y el nivel socioeconómico como predisponentes del embarazo, la mayoría de los estudios a este respecto son de carácter descriptivo y correlacional, más que predictivos.
} 
Más aún, en el trabajo realizado por Molina et al., al hacer un seguimiento después del parto, alrededor del $40 \%$ de las menores, seguía sin el uso de métodos anticonceptivos.

Estos hechos sumados a que menos del $10 \%$ de los adolescentes han recibido alguna educación sexual sistemática antes de los 13 años, teniendo inicio sexual antes de los 15 años, constituyen un panorama preocupante. (Gottsegen \& Philliber, 2001)

Éstos hallazgos están muy probablemente asociados a la fábula personal característica de la adolescente, del tipo "esto no me va a pasar a mi" (Flick, cit. en Desmond, 1994; Elkind cit. en Papalia y Wendkos, 1998), y que ha sido encontrado frecuentemente asociado al embarazo adolescente (Molina et al., 2001; Varas et al., 1999).

\section{Factores familiares}

Dentro de los factores familiares, se ha mostrado que el involucramiento paterno actúa disminuyendo en sus hijos conductas de riesgo (Scaramella et al., 1998). Las hijas de padres que muestran una mayor preocupación por quién acompaña a sus hijas en las salidas, y respecto a las fiestas, perciben mayor apoyo social (Castro y Matamala, 1996).

En esta misma línea, en investigaciones realizadas en Ecuador, E.E.U.U. y otros países; se observó que las adolescentes embarazadas reportaban menor calidad en la comunicación familiar, que sus pares no embarazadas (Arnold, Smith, Harrison \& Springer, 2000; Corcoran et al. 2000; Guijarro et al. 1999; Issler, 2001). En un estudio realizado en la $8^{\circ}$ región, la mayoría de las adolescentes embarazadas describió la relación con su madre como distante (Brandell y Cifuentes, 2000).

En el ámbito de la trasmisión transgeneracional, se ha observado que tanto, en zonas rurales como urbanas, las madres adolescentes provenían de familias cuyas progenitoras también lo habían sido en su mayoría (Molina et al., 1992; Porras, 1994; Short y Slusher, 1994). Se ha reportado además mayor porcentaje de padres alcohólicos en las adolescentes embarazadas (Arriagada et al.,1991; Molina et al., 1992; Rojas, 1991), y que provienen de familias numerosas (Molina et al., 1992).

Además se han planteado otras características que se pueden asociar a algún grado de disfunción familiar, como son, relaciones intrafamiliares complicadas y/o con violencia (Short y Slusher, 1994; Vives et al., 1992) y ausencia de uno o de los dos padres en el hogar (Brandell y Cifuentes, 2000, Florenzano, 1997; Guijarro et al., 1999; Issler, 2001; Molina et al., 1992; Medora, \& Von der Hellen, 1997; Rojas, 1991; Short \& Slusher, 1994). También el aumento de las discusiones entre los padres o problemas con los hermanos fueron observados como factores predisponentes al embarazo precoz (Records, 1993).

También se ha encontrado, que embarazadas que puntuaban altos niveles de estrés en sus familias de origen, consideraban sólo como moderadamente estresante el embarazo en si mismo, en relación a otros estresores. Esto puede ayudar a entender cómo es que el embarazo puede llegar a ser visto como una salida de relaciones familiares conflictivas (Ravert \& Martín, 1997).

En términos generales, una mayor disfuncionalidad familiar se ha asociado tanto al riesgo de embarazo, como al embarazo en sí mismo (Burrows, Rosales, Ayalo \& Muzzo, 1994; Medora, \& Von der Hellen, 1997; Varas et al., 1999).

\section{Apoyo social percibido}

Se han observado más bajos estándares de calidad y cantidad de relaciones sociales (Arriagada et al., 1991) en las embarazadas adolescentes, quienes además difícilmente encuentran apoyo para sus problemas tanto fuera como dentro de la familia (Guijarro et al., 1999). 
Arriagada et al. (1991) en una investigación realizada en Concepción, encontraron diferencias significativas entre grupos de embarazadas y no embarazadas en cuanto a la pertenencia a alguna entidad religiosa, participando mucho más las no embarazadas, lo que también se observó en Ecuador (Guijarro et al., 1999) y en E.E.U.U. (Arnold et al., 2000)

Por una parte, una buena calidad de apoyo por parte de los padres, es un predictor de apoyo social familiar para las adolescentes (Arnold et al., 2000; Castro \& Matamala, 1996), y por otra el menor nivel educacional de los padres parece ser otro factor predisponente al embarazo adolescente (Arraigada et al., 1991; Ferrada et al., 2002; Guijarro et al., 1999). Estos dos elementos combinados se pueden asociar a lo encontrado por Castro y Matamala (1996) que muestran que a mayor nivel educacional de los padres, las adolescentes reciben mayor apoyo familiar.

En La Paz, Bolivia, estos hallazgos se confirmaron por Lipovsek, Mehryar, Zielinski, Magnani y Castro (2002), quienes en un estudio que comparaba embarazadas adolescentes con jóvenes no gestantes, encontraron que las embarazadas sentían menor apoyo de sus padres, y tenían mayores conflictos con ellos.

Si consideramos que a menudo el primer encuentro sexual se produce en su propia casa o en la de algún amigo cercano, el apoyo y la supervisión familiar son muy importantes (Arnold et al., 2000).

En resumen se afirma que las adolescentes embarazadas, perciben menor apoyo social (Salinas y Armengol, 1992), destacándose sentimientos de aislamiento y falta de interés en las relaciones grupales.

Toda esta evidencia tiende a avalar la hipótesis de que un mayor apoyo social estaría asociado a una menor probabilidad de embarazarse durante la adolescencia.

\section{Nivel intelectual}

En cuanto al nivel intelectual, Carrasco (1986, cit. en Arriagada et al., 1991), señaló que al comparar adolescentes embarazadas y no embarazadas, el grupo de embarazadas presentaba un CI significativamente menor que el grupo control. Arriagada et al. (1991), encontraron que las embarazadas adolescentes presentan un menor nivel de pensamiento lógico-abstracto con relación a sus pares no embarazadas. Sin embargo, Lartigue et al., (1992), en un estudio realizado con adolescentes embarazadas reportaron, inteligencia dentro de rasgos normales.

Como se señalaba anteriormente, esta discusión puede ser muy importante en la medida que el razonamiento abstracto permitiría, en teoría, la anticipación de las consecuencias negativas que tiene un embarazo. Por ejemplo, en Concepción, Molina et al. (2001), encontraron que la justificación para no utilizar métodos anticonceptivos en un grupo de adolescentes embarazadas menores de 15 años, era el pensar "que ellas no iban a quedar embarazadas".

Es interesante señalar, que muchos de los programas de prevención que se realizan recientemente en E.E.U.U., pretenden considerar este tipo de pensamiento mágico y la etapa del desarrollo del razonamiento en que se encuentran las jóvenes, para diseñar estrategias de intervención, argumentando que el reconocimiento de los elementos del estado operacional formal de Piaget, puede ser útil para comprender la conducta contraceptiva de las adolescentes (Out \& Lafreniere, 2001).

Complementando lo anterior se encuentra una investigación, realizada por Medora y Von der Hellen (1997), con un grupo de madres adolescentes en California, y con un grupo control de jóvenes no madres, quienes encontraron que las primeras presentaban niveles significativamente más altos de romanticismo que el grupo control, teniendo este último percepciones más realistas con respecto a la maternidad, el amor y el matrimonio. 


\section{Autoestima}

Respecto a la autoestima se ha advertido información contradictoria. Short y Slusher (1994), plantean que las adolescentes embarazadas poseen un pobre autoconcepto de sí mismas, lo que también fue visto por Salinas y Armengol (1992), encontrando un bajo nivel de autoconcepto general, poca confianza en sí mismas, sentimientos ansiosos y depresivos, y además por Restrepo (1991), quién planteó la centralidad que tiene en el embarazo adolescente la baja autoestima. Siguiendo esta línea Lartigue et al. (1992) observaron puntajes en el límite más bajo de la normalidad en la variable autoconcepto. Esto también ha sido reportado en Bolivia (Lipovsek et al., 2002).

En Chile, en un grupo compuesto de embarazadas y menores en riesgo de embarazo, se encontraron bajos niveles de autoestima (Burrows et al., 1994). Apoyando lo anterior, estaría la investigación de Corona \& Tidwell (1999) con adolescentes, realizada en Estados Unidos, en la que se encontró, que algunas madres adolescentes se embarazaban intencionalmente porque siempre habían querido tener un hijo propio o alguien a quién amar, o como un medio para mantener a la pareja, lo que podría asociarse a una baja autoestima. Esto también, es corroborado por otros hallazgos en Estados Unidos y México (Lagana, 1999).

En Chile, se ha reportado que de un grupo de 60 adolescentes embarazadas o en puerperio, el 37\% manifestó haber planificado su embarazo ya sea por consolidar el vínculo de pareja o por el sentimiento de soledad (Vega, Gallegos \& Varela, 1999). Esto también fue señalado por Rojas (1991) como un camino en la búsqueda de la estabilidad emocional.

Sin embargo, existen otros autores que sostienen que no hay relación entre la autoestima y el embarazo adolescente (Medora \& Von der Hellen, 2000). Dentro de esta tendencia, está la investigación realizada en la ciudad de Concepción, donde no se encontraron diferencias significativas de autoestima entre jóvenes encinta y no embarazadas (Arriagada et al., 1991).

Por otra parte, en México, se encontró que en adolescentes embarazadas, el $54 \%$ de ellas tenía una autovaloración normal, mientras que un $46 \%$ mostraba una autoestima "narcisista", tendiendo a valorarse en exceso y a interesarse sólo en su persona (Gutierrez, Pascacio, De la Cruz y Carrasco, 2002).

\section{Depresión}

La depresión como factor asociado al embarazo adolescente no está claro si puede ubicarse como factor predisponente, ya que existen investigaciones que así lo señalan (Horwitz et al., 1991, cit. en Barnet, Joffe, Duggan, Wilson \& Repke, 1996), o como consecuencia de este. No obstante, en relación a este aspecto se ha encontrado la siguiente información.

Se han hallado mayores niveles de depresión en las adolescentes encinta (Guijarro et al., 1999; Restrepo, 1991). También se han encontrado hallazgos similares, en investigaciones realizadas en México con adolescentes embarazadas (Lartigue et al., 1992; Salinas \& Armengol, 1992).

En Estados Unidos, en un estudio que consideraba madres adolescentes, se encontraron niveles superiores al promedio para depresión en el 53\% de las menores (Brage, Elek \& Campbell-Grossman, 2000).

Complementando todo lo anterior, en un estudio realizado en Chile con embarazadas de diversas edades, quienes presentaban mayor sintomatología depresiva eran las adolescentes entre 15 y 19 años (Millán, Yevenez, Galvez \& Bahamonde, 1990). Así mismo, estos investigadores encontraron sentimientos de inseguridad, culpa y la tendencia a apartarse del grupo social. 
Barnet et al. (1996), encontraron que los niveles de depresión disminuyeron en el periodo de postparto, entre las jóvenes que estaban deprimidas previamente, y entre las que no lo estaban, se halló un aumento de estos niveles. Troutman y Cutrona (1990) encuentran que los síntomas depresivos continuaron hasta 6 semanas después del parto si no hay una intervención adecuada.

Al analizar los hallazgos en torno a la variable depresión es importante recordar lo encontrado por Lartigue et al. (1992), quienes señalaron que las adolescentes perciben menos apoyo de su pareja que de su familia de origen (Molina et al, 1997), aún cuando reportan necesitarlo, y lo indicado por Barnet et al. (1996), que puntualizaron que la falta de apoyo social aumenta las probabilidades de que aparezcan síntomas depresivos.

Es evidente que en muchos casos el proyecto de vida de estas adolescentes se ve frustrado (Muñoz et al., 2001), lo que también podría asociarse a sintomatología depresiva.

No obstante, Troutman y Cutrona (1990), no encontraron diferencias significativas en depresión entre un grupo de adolescentes embarazadas y posteriormente madres, y un grupo control con las mismas características sociodemográficas y en una investigación realizada en E.E.U.U., con adolescentes en el tercer trimestre de embarazo, se verificó que en la medida que el embarazo avanzaba, los síntomas depresivos disminuían progresivamente (Barnet et al., 1996).

El analizar factores psicológicos y psicosociales en mujeres embarazadas menores de 15 años se hace particularmente necesario, por los escasos estudios al respecto, por la necesidad de profundizar el conocimiento existente, y especialmente por las singularidades que esta etapa del desarrollo tiene, y que son distintas al grupo etáreo mayor de 16 años.

\section{Objetivos}

Analizar si existen o no diferencias entre madres adolescentes primigestas menores de 15 años, de NSE bajo, y madres primigestas entre 20 y 34 años del mismo nivel socioeconómico, con respecto a sus características psicológicas.

Analizar si existen o no diferencias entre madres primigestas adolescentes menores de 15 años, de NSE bajo y adolescentes no madres de la misma edad, del mismo nivel socioeconómico, con respecto a sus características psicológicas.

\section{Metodología}

\section{Hipótesis}

1. Las madres adultas primigestas entre 20 y 34 años, de NSE bajo, presentan significativamente mayores niveles de apoyo social percibido que las adolescentes madres primigestas menores de 15 años y de igual NSE.

2. Las madres adultas primigestas entre 20 y 34 años, de NSE bajo, presentan significativamente mayor nivel intelectual que las adolescentes madres primigestas menores de 15 años y de igual NSE.

3. Las madres adultas primigestas entre $20 \mathrm{y}$ 34 años, de NSE bajo, presentan significativamente mayores niveles de autoestima que las adolescentes madres primigestas menores de 15 años y de igual NSE.

4. Las adolescentes no madres menores de 15 años, de NSE bajo, presentan significativamente mayores niveles de apoyo social percibido que las adolescentes madres de la misma edad y NSE.

5. Las adolescentes no madres menores de 15 años, de NSE bajo, presentan significativamente mayor sintomatología de- 
presiva que las adolescentes madres de la misma edad y NSE.

6. Las adolescentes no madres menores de 15 años, de NSE bajo, presentan significativamente mayor nivel intelectual que las adolescentes madres de la misma edad y NSE.

7. Las adolescentes no madres menores de 15 años, de NSE bajo, presentan significativamente mayores niveles de autoestima que las adolescentes madres de la misma edad y NSE.

\section{Muestra}

El muestreo fue de tipo temporal. La muestra correspondió a un año (2001) y correspondió a 46 adolescentes primigestas que tuvieron su parto durante ese período de tiempo. De estas 46 niñas, se pudo encuestar a 41 , siendo entonces este el $n$ valido.

Derivado de lo anterior, la muestra estuvo constituida por las madres primigestas menores de 15 años, cuyos partos ocurrieron en el servicio de obstetricia y ginecología del Hospital Guillermo Grant Benavente durante el año 2001.

El primer grupo control estuvo constituido por un numero equivalente de madres primigestas, entre 20 y 34 años del mismo
NSE, cuyos partos ocurrieron en el servicio de obstetricia y ginecología del Hospital Guillermo Grant Benavente, durante el año 2001. Así, por cada madre adolescente que se atendía de parto en el Hospital Guillermo Grant Benavente, se elegía al azar alguna mujer entre 20 y 34 años que fuese primigesta y que se hubiese atendido de parto ese mismo día.

El segundo grupo control estuvo constituido por una muestra obtenida por accesibilidad, de adolescentes no madres ni embarazadas, de un rango de edad equivalente y mismo NSE, alumnas de liceos y escuelas públicas de la ciudad de Concepción y sus alrededores. Este grupo fue detectado, a través de informantes claves, del Servicio de Salud Concepción y la Dirección de Educación de Chiguayante. ${ }^{5}$

\section{Instrumentos}

Para este estudio las variables consideradas fueron apoyo social percibido, depresión, coeficiente intelectual, y autoestima.

Para medir cada una de ellas se utilizó un set de instrumentos. A continuación se les enumera, mencionando el autor y los datos de confiabilidad y validez originales y para Chile.

5 Como el segundo grupo control no fue elegido probabilísticamente, estrictamente no se deberían usar métodos de análisis estadístico paramétrico. Sin embargo, éstos se utilizaron a partir de los objetivos de la investigación, y teniendo presente que las inferencias se pueden hacer, sólo a una población con características similares a las de la muestra, lo que sería el objetivo de este estudio. 
Tabla 1

\begin{tabular}{|c|c|c|c|c|c|}
\hline Escala & Autor & \begin{tabular}{|c|} 
Validez \\
Original
\end{tabular} & $\begin{array}{l}\text { Confia- } \\
\text { bilidad } \\
\text { Original }\end{array}$ & Validez Chile & Confiabilidad Chile \\
\hline $\begin{array}{l}\text { Escala de Apoyo } \\
\text { Social Percibido. }\end{array}$ & $\begin{array}{l}\text { Zimet et al. } \\
\text { (s/f, en Blumenthal } \\
\text { et al., 1987). }\end{array}$ & 0.85 & 0.88 & $\begin{array}{l}0.80 \\
\left(D^{\prime} \text { Appolonio }\right. \\
\text { y cols., 2000). }\end{array}$ & 0.87 \\
\hline $\begin{array}{l}\text { Subescala de Sintomas } \\
\text { Depresivos, de la Escala } \\
\text { de Salud Mental. }\end{array}$ & $\begin{array}{l}\text { Alvarado et al. } \\
\text { (1991). }\end{array}$ & & & $0.70-0.95$ & 0.93 \\
\hline $\begin{array}{l}\text { CDI. Children's } \\
\text { Depression Inventory. } \\
\text { Adaptación para la } \\
\text { población española. }\end{array}$ & $\begin{array}{l}\text { Del Barrio, } \\
\text { Moreno-Rosset } \\
\text { y López- } \\
\text { Martinez, } 1999 .\end{array}$ & 0.84 & $* * *$ & $\begin{array}{l}0.82 \\
\text { (Venegas et al., } \\
\text { 2001). }\end{array}$ & 0.81 \\
\hline $\begin{array}{l}\text { Test de Habilidades } \\
\text { Mentales Primarias. }\end{array}$ & $\begin{array}{l}\text { Manzi y Rosas, } \\
1985 .\end{array}$ & $* * *$ & $* * *$ & $* * *$ & $\begin{array}{l}\text { Significado Verbal, } \\
\text { 0.91, series } \\
\text { numéricas, 0.77, } \\
\text { Series de Letras, } \\
\text { 0.78, Agrupación } \\
\text { de Palabras, 0.79, } \\
\text { Relaciones Espaciales, } \\
\text { 0.83 (Manzi y Rosas, } \\
\text { 1985). }\end{array}$ \\
\hline $\begin{array}{l}\text { Escala de Autoestima } \\
\text { de Coopersmith. }\end{array}$ & $\begin{array}{l}\text { Validación hecha en } \\
\text { Chile por Brikmann } \\
\text { y Garcés (1999), } \\
\text { para adultos, y por } \\
\text { Brinkmann, Segure } \\
\text { y Solar (1989), para } \\
\text { adolescentes y niños. }\end{array}$ & $* * *$ & $* * *$ & $\begin{array}{l}\text { Adolescentes. } \\
\text { Validez, } \\
\text { correlacionando } \\
\text { el test de } \\
\text { personalidad } \\
\text { de California, } \\
\text { significativas } \\
\text { al } p=0.01 \text {. }\end{array}$ & $\begin{array}{l}\text { Adolescentes: } 0.87 \\
\text { (KR-20 para las } \\
\text { cinco escalas). } \\
\text { (Brinkmann, Segure } \\
\text { y Solar, 1989) } \\
\text { Adultos: } 0.85 \\
\text { (Brinkmann y } \\
\text { Garcés, 1999). }\end{array}$ \\
\hline
\end{tabular}

\section{Resultados}

\section{Descriptivos.}

A continuación se presentan los estadísticos descriptivos de las variables que se consideraron en el presente estudio, para el grupo experimental. 
Tabla 2

\begin{tabular}{|l|c|c|c|c|}
\hline & Media & Desviación Estándar & Valor Mínimo & Valor Máximo \\
\hline Edad & 14.59 & 0.63 & 13 & 15 \\
\hline Edad Madre Nacimiento & 14.29 & 0.68 & 12 & 15 \\
\hline Edad Hijo (meses) & 3.95 & 2.05 & 0 & 8 \\
\hline Años estudio & 7.24 & 1.71 & 4 & 12 \\
\hline Apoyo Social General & 64.80 & 10.61 & 47 & 84 \\
\hline Apoyo Social Amigos & 18.59 & 6.80 & 4 & 28 \\
\hline Apoyo Social Familia & 20.83 & 6.16 & 5 & 28 \\
\hline Apoyo Social Otros & 25.66 & 2.58 & 18 & 33 \\
\hline Depresión & 13.51 & 5.87 & 3 & 117 \\
\hline CI & 76.85 & 14.28 & 50 & 80 \\
\hline Autoestima & 51.54 & 12.13 & 31 & 62 \\
\hline Autoestima Social & 48.76 & 8.24 & 27 & 78 \\
\hline Autoestima Escolar & 55.71 & 12.06 & 30 & 70 \\
\hline Autoestima Familiar & 48.17 & 9.79 & 32 & 65 \\
\hline Autoestima General & 47.2 & 9.66 & 30 & 33 \\
\hline
\end{tabular}

\section{Estadística bivariada}

A continuación se informan los resultados derivados de la comparación entre los tres grupos incluidos en la investigación, en torno a las variables señaladas como relevantes según los objetivos e hipótesis planteadas. Este análisis para apoyo social, CI y autoestima, se llevó a cabo mediante Análisis de Varianza, para los tres grupos. Luego se expone el resultado de la prueba post hoc de Scheffe, que muestra si existen diferencias significativas entre el grupo objetivo y cada uno de los grupos de control.
En el caso de la variable depresión, se muestra el resultado de la prueba $T$, él que fue dividido por dos por ser la hipótesis direccional.

\section{Apoyo Social Percibido}

Es pertinente indicar que en torno a esta variable, es necesario poner atención no sólo al Apoyo Social General, sino que a cada una de las subescalas que lo componen, ya que esto permitirá profundizar el análisis de los datos.

Tabla 3

Resultado de la Prueba Anova para Apoyo Social Percibido

\begin{tabular}{|l|c|c|c|c|}
\cline { 2 - 5 } \multicolumn{1}{c|}{} & $\begin{array}{c}\text { Media de los } \\
\text { cuadrados }\end{array}$ & $\begin{array}{c}\text { Grados de } \\
\text { libertad }\end{array}$ & F & Significancia \\
\hline Apoyo Social Total & 17,809 & 2 & 0,081 & 0,922 \\
\hline Apoyo Social Amigos & 357,140 & 2 & 7,034 & 0,001 \\
\hline Apoyo Social Familia & 87,222 & 2 & 1,705 & 0,186 \\
\hline Apoyo Social Otros & 134,458 & 2 & 5,736 & $0,0046^{* *}$ \\
\hline
\end{tabular}

${ }^{* *} p<0.01$ 
Tabla 4

Resultados del Test Posthoc de Scheffe para Apoyo Social Percibido

\begin{tabular}{|l|c|c|c|c|}
\cline { 2 - 5 } \multicolumn{1}{c|}{} & $\begin{array}{c}\text { Apoyo Social } \\
\text { Total }\end{array}$ & $\begin{array}{c}\text { Apoyo Social } \\
\text { Amigos }\end{array}$ & $\begin{array}{c}\text { Apoyo Social } \\
\text { Familia }\end{array}$ & $\begin{array}{c}\text { Apoyo Social } \\
\text { Otros }\end{array}$ \\
\hline $\begin{array}{l}\text { Diferencia Entre Medias Objetivo } \\
\text { (Grupo 2) - Control (Grupo 1) }\end{array}$ & 1,317 & 0,439 & $-0,732$ & 1,61 \\
\hline $\begin{array}{l}\text { pentre Objetivo (Grupo 2) y Adultas } \\
\text { (Grupo 1), para una cola. }\end{array}$ & 0,46 & 0,48 & 0,45 & 0,17 \\
\hline $\begin{array}{l}\text { Diferencia Entre Medias Objetivo } \\
\text { (Grupo 2) - Control (Grupo 3) }\end{array}$ & 0,61 & $-5,109$ & 2,19 & 3,742 \\
\hline $\begin{array}{l}\text { pentre Objetivo (Grupo 2) y } \\
\text { Adolescentes (Grupo 3), para una cola. }\end{array}$ & 0,49 & $0,0045 * *$ & 0,21 & $0,002 * *$ \\
\hline
\end{tabular}

Obs. El signo negativo de la diferencia entre las medias, indica que es el grupo Control Adolescentes no madres el que presentó significativamente Mayor Apoyo Social de Amigos en relación al grupo de Adolescentes Madres ** $p<0.01$

Como se puede observar, si bien, no se dan diferencias significativas en el apoyo social percibido general, si las hay en el apoyo social percibido entre adolescentes madres y no madres con relación a los amigos, y a los otros; percibiendo significativamente mayor apoyo social de los amigos las adolescentes no madres; y percibiendo significativamente mayor apoyo social de otros las madres adolescentes.
Si bien, no está dentro de las hipótesis la comparación entre las madres adultas y las adolescentes no madres, en la variable apoyo social percibido con relación a los amigos, se revisó la significancia de éstas por considerarlo pertinente (como se discutirá en las conclusiones finales), encontrando lo informado en la Tabla 8.

Tabla 5

Resultados del Test Posthoc de Scheffe para Apoyo Social Percibido Amigos entre el grupo de adolescentes no madres y el grupo de adultas madres.

\begin{tabular}{|c|c|c|}
\cline { 2 - 3 } \multicolumn{1}{c|}{} & $\begin{array}{c}\text { Diferencia Entre Adolescentes } \\
\text { no Madres (Grupo 3) - } \\
\text { Adultas (Grupo 1) }\end{array}$ & $\begin{array}{c}\text { Significancia entre adolescentes } \\
\text { no Madres (Grupo 3) y } \\
\text { Adultas (Grupo 1) para una cola. }\end{array}$ \\
\hline Apoyo Social Amigos & 5,548 & $0.002^{* *}$ \\
\hline
\end{tabular}

${ }^{* *} p<0.01$

Al dividirlo por dos, el valor p queda en $0,002^{* *}$, lo que implica que existe diferencia significativa en la percepción de apoyo social de amigos de parte de las adolescentes no madres en relación con las madres adultas, a favor de las primeras.

\subsubsection{Depresión}

En esta variable se informará sólo la comparación, mediante la prueba $\mathrm{T}$, entre el grupo 2 y el grupo 3, con quienes se utilizó la misma prueba, el CDI. Luego se muestra el resultado dividido por dos por ser la hipótesis direccional. 
Con el grupo 1 no es posible hacer una comparación real, ya que a ellas se les apli- có la subescala de depresión de Alvarado et al. (1991)

Tabla 6

Prueba $t$ de student entre el grupo de adolescentes madres y adolescentes no madres para la variable depresión

\begin{tabular}{|l|c|c|}
\hline Depresión & $\boldsymbol{t}$ & Valor p para una cola. \\
\hline Varianzas Iguales Asumidas & -1.89 & $0.032^{*}$ \\
\hline
\end{tabular}

Obs: el signo negativo de tindica la diferencia a favor del grupo de adolescentes no embarazadas.

Al dividir por dos el valor p para dos colas, queda en $0,032 *$, lo que implica que existe diferencia significativa en la sintomatología depresiva entre el grupo objetivo madres adolescentes y las adolescentes no madres. No obstante la diferencia es a favor de este último grupo y no como se propone en la hipótesis número 5.

\subsubsection{Coeficiente Intelectual (CI)}

Con respecto a la comparación en el Coeficiente Intelectual entre el grupo experimental y los grupos control, se puede afirmar que, como se puede apreciar en la tabla 10 , existen diferencias significativas entre el grupo objetivo, y el control 2 (Adolescentes no madres), presentando más alto CI el último. No se encontraron diferencias significativas con el Grupo 1.

Tabla 7

Resultado de la Prueba Anova entre los tres grupos para Coeficiente Intelectual.

\begin{tabular}{|c|c|c|c|c|}
\cline { 2 - 5 } \multicolumn{1}{c|}{} & $\begin{array}{c}\text { Media de los } \\
\text { cuadrados }\end{array}$ & $\begin{array}{c}\text { Grados de } \\
\text { libertad }\end{array}$ & $\boldsymbol{F}$ & Significancia \\
\hline Coeficiente Intelectual & 601,942 & 2 & 3,513 & $0,033^{*}$ \\
\hline
\end{tabular}

${ }^{*} p<0.05$

Tabla 8

Resultados del Test Posthoc de Scheffe para Coeficiente Intelectual

\begin{tabular}{|l|c|}
\cline { 2 - 2 } \multicolumn{1}{c|}{} & Coeficiente Intelectual \\
\hline Diferencia Entre Medias Objetivo (Grupo 2) - Control (Grupo 1) & $-3,61$ \\
\hline pentre Objetivo (Grupo 2) y Adultas (Grupo 1), para una cola. & 0,23 \\
\hline Diferencia Entre Medias Objetivo (Grupo 2) - Control (Grupo 3) & $-7,924$ \\
\hline pentre Objetivo (Grupo 2) y Adolescentes (Grupo 3), para una cola. & $0,017^{*}$ \\
\hline
\end{tabular}

${ }^{*} p<0.05$

Como se puede observar no existen diferencias significativas en el CI entre las madres adultas y las madres adolescentes menores de 15 años. Si se encuentran entre las adolescentes no madres y las madres menores de 15 años, siendo esta diferencia a favor de las primeras $(\mathrm{p}=0.017)$. 


\section{Autoestima}

Es pertinente indicar que en torno a esta variable, es necesario atender no sólo a la
Autoestima Total, sino que a cada una de las subescalas que la componen, que son autoestima social, escolar, familiar y general.

Tabla 9

Resultado de la Prueba Anova entre los tres grupos para autoestima

\begin{tabular}{|l|c|c|c|c|}
\cline { 2 - 5 } \multicolumn{1}{c|}{} & $\begin{array}{c}\text { Media de } \\
\text { los cuadrados }\end{array}$ & $\begin{array}{c}\text { Grados de } \\
\text { Libertad }\end{array}$ & $\boldsymbol{F}$ & Significancia \\
\hline Autoestima total & 308,372 & 2 & 1.824 & 0,166 \\
\hline Autoestima Social & 335,444 & 2 & 3,352 & $0,038^{*}$ \\
\hline Autoestima escolar & 390,932 & 2 & 2,428 & 0,093 \\
\hline Autoestima Familiar & 413,963 & 2 & 3,26 & $0,042 *$ \\
\hline Autoestima General & 230,392 & 2 & 2,766 & 0,067 \\
\hline
\end{tabular}

${ }^{*} p<0.05$

Tabla 10

Resultados del Test Posthoc de Scheffe para Autoestima

\begin{tabular}{|c|c|c|c|c|c|}
\hline & $\begin{array}{c}\text { Autoestima } \\
\text { Total }\end{array}$ & $\begin{array}{c}\text { Autoestima } \\
\text { Social }\end{array}$ & $\begin{array}{c}\text { Autoestima } \\
\text { Escolar }\end{array}$ & $\begin{array}{c}\text { Autoestima } \\
\text { Familiar }\end{array}$ & $\begin{array}{c}\text { Autoestima } \\
\text { General }\end{array}$ \\
\hline $\begin{array}{l}\text { Diferencia Entre Medias } \\
\text { Objetivo (Grupo 2) - } \\
\text { Control (Grupo 1) }\end{array}$ & 0,146 & 5,146 & 5,024 & $-2,927$ & $-0,878$ \\
\hline $\begin{array}{l}\text { p entre Objetivo (Grupo 2) } \\
\text { y Adultas (Grupo 1), } \\
\text { para una cola. }\end{array}$ & 0,5 & $0,036^{*}$ & 0,11 & 0,25 & 0,46 \\
\hline $\begin{array}{l}\text { Diferencia Entre Medias } \\
\text { Objetivo (Grupo 2) - } \\
\text { Control (Grupo 3) }\end{array}$ & 5,037 & 0,312 & 5,763 & 3,643 & 3,779 \\
\hline $\begin{array}{l}\text { p entre Objetivo (Grupo 2) } \\
\text { y Adolescentes (Grupo 3), } \\
\text { para una cola. }\end{array}$ & 0,12 & 0,5 & 0,07 & 0,18 & 0,099 \\
\hline
\end{tabular}

Existen diferencias significativa en autoestima social, entre el grupo objetivo y el control 1 (madres adultas), a favor de las primeras y significancia débil en las diferencias de autoestima escolar y autoestima general entre el grupo objetivo y el control 2 (adolescentes no madres), presentando las primeras mas altos índices en estas dos variables. 


\section{Análisis y Discusión}

Se debe iniciar esta discusión, señalando que los resultados y conclusiones obtenidas en la presente investigación tienen que ser entendidas en el marco del escaso conocimiento que se tiene en torno a las características de este problema en menores de 15 años. Por lo tanto, hay dos alcances básicos que destacar.

Primero, que los datos entregados por la estadística descriptiva constituyen en sí mismos información relevante para futuras investigaciones y para contrastar con hallazgos previos. Segundo, que el hecho de que exista poca investigación anterior en este segmento etáreo, hace que los datos de este informe deban ser valorados no sólo a partir de la corroboración o refutación de las hipótesis, sino que un original aporte al conocimiento en el tema.

En términos generales, mientras que la media de las madres adultas, en el momento de la entrevista es de 25 años aproximadamente y al nacimiento de su primer hijo tenían 24.6 años, la media de las madres adolescentes, en el momento de la entrevista, es de 14.6 años. Al nacimiento de su primer hijo tenían 14.3 años. En este grupo el embarazo se produjo a los 14 años como máximo.

Es evidente que esta diferencia etárea no sólo refleja distinciones en el periodo de desarrollo con relación a las madres adultas, sino que también la baja edad de las niñas corresponde a la transición entre la primera y segunda etapa de la adolescencia, donde recién los cambios propios de esta edad están ocurriendo. Si toda la adolescencia en un periodo de transformación entre la infancia y la adultez, los sujetos de este estudio se encuentran mucho más cerca de la primera, lo que implica, que recién han comenzado a cumplir las primeras tareas de este período (Florenzano,
1997). Esto sería muy distinto si tuvieran 17 o 18 años, donde no sólo se están resolviendo muchas de las tareas relacionadas con la identidad (Erikson, 2000) sino que además a menudo están terminado la enseñanza media.

Las adolescentes madres mayoritariamente son solteras (40 de 41). Esto contribuye a corroborar lo señalado por el INE (1998) que indica que en Chile, el 95\% de los hijos de menores de 15 años son nacidos fuera del matrimonio.

De todas formas debemos entender que el matrimonio a esta edad no constituye por si mismo un seguro de felicidad o bienestar para la madre o el bebé, en la medida que como se señalaba anteriormente, no están dadas aún las condiciones necesarias en términos evolutivos para la consolidación de un núcleo familiar que otorgue garantías afectivas y de satisfacción de necesidades básicas.

Como otro elemento importante a destacar, antes de analizar los resultados por variable, se debe mencionar primero, que los hijos/ as de las adultas tenían 4 meses aproximadamente en el momento de la entrevista, al igual que los hijos/as de las adolescentes. Por lo tanto se pueden comparar las diferencias en las variables medidas, en tanto influidas por sus experiencias como madres primerizas.

En segundo lugar, como no es un estudio longitudinal, es difícil saber el nivel que alcanzaban las variables previo a la medición, en particular, antes o durante el embarazo. Esto se volverá a retomar al final de las presentes conclusiones.

Por último, el hecho de que las adolescentes madres y las adolescentes no madres tengan el mismo nivel educativo ${ }^{6}$, permite comparar ambos grupos en las variables analizadas.

6 No se encontraron diferencias significativas, siendo el p arrojado por el test Post-Hoc de Scheffe de 0.97, luego de haber analizado esta variable con la prueba ANOVA. Estos resultados no son informados en la presente investigación por no estar incluidos en los objetivos ni en las hipótesis centrales de ésta. 


\section{Apoyo social percibido}

Para comenzar es interesante observar que aún cuando no existen diferencias significativas en el apoyo social percibido general entre los tres grupos, lo que no concuerda con lo expuesto por Salinas y Armengol (1992), si las hay en la subescala amigos y en la subescala otros; no así en la subescala familiar, lo que contradiría a lo encontrado por Guijarro et al. (1999).

Formalmente, entonces se deberían rechazar las hipótesis 1 y 4 .

No obstante, el siguiente análisis enriquece la discusión. En las variables apoyo social percibido de amigos, y apoyo social percibido de otros, las adolescentes madres se comportan de manera muy similar a las madres adultas, no como sus pares de edad.

Las adolescentes no madres perciben significativamente mayor apoyo social por parte de sus amigos, con relación a sus coetáneas y a las madres adultas, lo que se puede deber a su inserción a nivel escolar lo que quizás, podría operar como factor protector; esto podría relacionarse con los menores niveles de calidad y cantidad de relaciones sociales, encontrados por Arriagada et al. (1991), con lo encontrado en Costa Rica por Rojas (1991) y con lo hallado por Salinas y Armengol (1992). Es importante señalar que no contar con el apoyo del grupo de pares en esta edad es mucho más dañino que en la adultez, por la centralidad que estos tienen en la adolescencia. De hecho Lipovsek et al. (2002), señalan explícitamente que las niñas que contaban menos con su grupo de pares, tienen muchas más probabilidades de embarazarse.

Sin embargo, en la subescala otros, las madres adolescentes perciben significativamente mayor apoyo social de parte de otros que no son familia ni amigos, concordando sólo en parte con lo encontrado en Ecuador (con un diseño de investigación similar al utilizado en la presente investigación) por Guijarro et al. (1999). Sería interesante profundizar quienes son estos otros. Se podría pensar que son redes de su sector, o la iglesia o bien el sistema de salud. Esto señala una tarea futura para otra investigación, ya que puede ser muy relevante en la salud mental de estas jóvenes y se debería considerar para intervenciones con ellas.

\section{Depresión}

Con respecto a determinar si alguno de los grupos presenta índices de depresión importantes, sólo es posible contestar esta pregunta con relación a las madres adultas, ya que es la única escala que cuenta con puntajes de corte para Chile. La escala CDI sólo cuenta con puntajes para la población española, y en el presente estudio se utilizó solamente con fines de comparación entre las adolescentes.

Las mujeres adultas presentan un puntaje promedio en la subescala de depresión de 9.05 lo que las ubica, si se siguen los parámetros de validación de Alvarado et al. (1991), dentro de los parámetros normales.

Al comparar a los dos grupos de adolescentes, existen diferencias significativas entre las madres adolescentes y las adolescentes no madres, siendo estas últimas las que presentan mayor grado de depresión $(\mathrm{p}=$ 0.032 ). Sin embargo, esta diferencia opera a favor de las adolescentes no madres, rechazándose la hipótesis 6.

Por una parte, esto es relevante ya que al ser los dos grupos similares (en edad, NSE y escolaridad), sería factible pensar que el sentimiento depresivo pueda ser un factor de riesgo y que al ser madres este disminuye, teniendo las menores un nuevo sentido de vida. Por lo tanto, sería de gran importancia trabajar con jóvenes que presenten sintomatología depresiva como una forma de prevenir el embarazo adolescente. 
Por otro lado, las autoras de la presente investigación consideran que la edad de la menores incluidas en la muestra incide en que no se encuentren mayores índices de depresión, ya que no existe aún una real percepción de la magnitud de la situación que están viviendo, por lo que esto contradice algunos hallazgos de la literatura en relación a adolescentes mayores (Brage et al., 2000; Millán et al., 1990), pero se aclara a la luz de lo encontrado por Medora y Von der Hellen (1997) quienes encuentran mayor romanticismo en las madres adolescentes en relación a sus pares no embarazadas ni madres, lo que apuntaría a una forma de razonamiento más infantil.

Sin embargo, al atenerse a lo informado por Barnet et al. (1994), en torno a que la sintomatología depresiva en adolescentes disminuye después del parto, se podría pensar que podría haber un porcentaje mayor de jóvenes que mostraban esta sintomatología en su período de embarazo.

\section{Coeficiente intelectual}

Las comparaciones entre el grupo experimental y los grupos control fueron hechas con la escala de Thurstone. Esta escala, como se señaló en la sección metodológica, ha sido utilizada en nuestro país con anterioridad con una muestra similar, y fue validada originalmente por Donoso et al. en 1983 (Pinto et al, 1991). Presenta ventajas prácticas en su aplicación en comparación con pruebas equivalentes.

Sin embargo, como se sabe, este instrumento clasifica a los sujetos en intervalos de CI más bajos que aquellos que les correspondería si hubiesen sido evaluados con la Escala WAIS o WISC. Esto permite entender los bajos puntajes obtenidos por todos los grupos, y no se debe concluir de forma apresurada que el nivel intelectual de la muestra es más bajo que la norma de la población general.
Por estas razones el énfasis en las conclusiones, está puesto en la comparación entre los grupos, que es lo planteado en la hipótesis de trabajo.

Así, en lo referente al nivel intelectual se aprecian diferencias significativas $(p=0.017)$ entre los dos grupos de adolescentes, teniendo más alto CI las adolescentes no madres. Esto concuerda con lo señalado por Arriagada et al. (1991), confirmando la hipótesis 5 . Si se considera el CI en términos de edad mental, se puede suponer que un mayor CI implica un mayor avance en las etapas del razonamiento cognitivo. Como se señalaba en el marco teórico, la capacidad de anticipación de las consecuencias de un embarazo no deseado, es más probable si se poseen las capacidades de previsión simbólica propias de un razonamiento más abstracto (Out \& Lafreniere, 2001).

Como se sabe existe una alta correlación entre el CI materno y el funcionamiento cognitivo de los hijos (Miller et al., 1996) y por lo tanto, se podría hipotetizar una transmisión transgeneracional de la limitación cognitiva. Con estos elementos se aclara la transmisión transgeneracional de la pobreza y la profunda dificultad de las jóvenes para encontrar un trabajo digno.

Con respecto a la hipótesis 2, no se encontraron diferencias significativas en el coeficiente intelectual entre las adolescentes madres y las madres adultas, debiendo esta por lo tanto ser rechazada. La interpretación de este hallazgo, es más compleja, ya que si bien en la literatura no se encontraron comparaciones específicas entre adolescentes madres y madres adultas, es razonable esperar que la postergación hasta la adultez del embarazo en mujeres de NSE bajo, implique un mayor nivel intelectual. Sin embargo, las autoras de la presente investigación, consideran que la ausencia de diferencias significativas podría originarse, en la falta de un entrenamiento regular en las destrezas cognitivas medidas por la prueba de 
Thurstone. Esta, que puede considerarse una limitación de la presente tesis, y que invita a una cuidadosa elección de instrumentos de evaluación de inteligencia, evidencia al mismo tiempo la escasez que existe en nuestro medio de pruebas que midan CI sin verse tan afectadas por el entrenamiento escolar.

Lo expuesto en el párrafo anterior, lleva a una reflexión acerca de los postulados de autores como Vigotsky y Ausubel (Pozo, 1997), quienes entienden el desarrollo cognitivo como inseparable del contexto social e instruccional.

\section{Autoestima}

Si bien todos los rangos de autoestima caen dentro la normalidad para los tres grupos, lo que apoyaría investigaciones como la de Arriagada et al. (1991) y la de Gutierrez et al. (2002), se observan ciertas diferencias en alguna de las subescalas del test de Coopersmith.

Como se mencionó, no aparecen diferencias significativas en la variable autoestima, medida con el puntaje total (autoestima total) en la Escala de Autoestima de Coopersmith. Estas diferencias no se encuentran ni entre el grupo objetivo y el grupo de madre adultas, rechazando por esto la hipótesis 3, ni entre el grupo objetivo y el grupo de adolescentes no madres, rechazando así la hipótesis 7.

No obstante lo anterior, al llevar a cabo un análisis por subescalas, se encontró que las madres adolescentes presentan una autoestima social significativamente más alta que las adultas $(p=0.036)$. La autoestima social es definida dentro de la escala de Coopersmith como el nivel de aceptación con que la persona valora sus conductas autodescriptivas en relación con sus pares (Brinkmann et al., 1989). Si se toma esta definición se puede especular que las madres adolescentes se evalúan más positivamente en sus competencias sociales, que las madres adultas.
Sin embargo, esto es poco concordante con los resultados obtenidos en la subescala de apoyo social percibido de amigos, donde no se encontraron diferencias significativas entre estos dos grupos. Por el contrario, presentaron medias casi equivalentes, siendo para las adolescentes madres de 18,59 y para las adultas 18,15. Es decir, las adolescentes madres se autoevalúan como más competentes en las relaciones con sus pares, aún cuando no encuentran apoyo en ellos. Esta incongruencia, se asocia con el pensamiento mágico ya descrito en la presente investigación, avalado por en el menor nivel intelectual de las menores en estudio.

La misma línea de análisis se da al comparar la autoestima escolar donde también se observa una diferencia significativa débil $(p=0.07)$, a favor de las madres adolescentes, en relación a las adolescentes no madres. Es decir estas jóvenes a pesar de presentar bajo CI, y en muchos casos haber desertado del sistema escolar, se perciben como más competentes en el ámbito académico que sus pares. Esto corroboraría lo señalado en el párrafo anterior.

Con respecto a la autoestima general, que corresponde al nivel de aceptación con que la persona valora sus conductas autodescriptivas (Brinkmann et al., 1989), existe una diferencia débilmente significativa, a favor de las madres adolescentes, en relación a las adolescentes no madres $(\mathrm{p}=0.09)$. Esta autoaceptación es concordante con el menor nivel de depresión encontrado en ellas, y respalda nuevamente la interpretación hecha por las autoras de la presente investigación en torno a que el pensamiento poco realista e infantil, es un elemento predominante en la vivencia de estas niñas.

Como se puede anticipar, la posibilidad de hacer predicciones a partir de los datos obtenidos en la presente investigación está limitado por el diseño de esta. El hecho de que sea un estudio transversal y no longitudinal hace muy difícil el determinar que factores se constituyen como gatillantes o de riesgo. 
Otra posibilidad sería utilizar un análisis multivariado para predecir el comportamiento de una variable, en este caso embarazo o no embarazo, a partir de otras. En la presente investigación, por el tamaño de la muestra sería poco viable utilizar un análisis multivariado, ya que el n debería ser cercano a 150 sujetos.

Si se adoptara la opción de un estudio longitudinal se debería pensar en varios años de investigación. En el caso de realizar un análisis multivariado, debido al tamaño del universo, se requeriría realizar un estudio en varias regiones del país paralelamente.

A pesar de estas dificultades, las autoras de la presente investigación, consideran que el hecho de haber ocupado instrumentos compuestos de subescalas tales como el Coopersmith y la Escala de Apoyo Social Percibido, fue un acierto, en la medida que permitió precisar relaciones que no habrían sido detectadas al usar escalas generales. Esto ayudó a visualizar con mayor claridad el complejo mundo de experiencia de la adolescente, pudiendo aislar elementos específicos que debieran ser reforzados en una intervención.

\section{Referencias Bibliográficas}

Alvarado, R., Vera, A., Toledo, M., Serrano, F., Gatica, C., NúÑez, C., Godoy, S., Soto, O., Alfaro, J. \& Asun, D. (1991). Validación de escalas para medir trastornos depresivos, por ansiedad y somatoformes. Cuadernos de Trabajo No2, Escuela de Psicología, Facultad de Ciencias Sociales, Universidad Diego Portales.

Arnold, E., Smith, T., Harrison, D. \& Springer, D. (2000) Adolescents' Knowledge and beliefs about pregnancy: the impact of "ENABL". Adolescence, 35, 485 - 498.

Arriagada, X., Bustamante, S., Carrasco, S., Cores, C. Espinoza, P. Hinostroza, M., Perez,
P. \& TORRes, C. (1991) Estudio comparativo de variables psicológicas de adolescentes embarazadas y no embarazadas. Seminario para optar al grado académico de Licenciado en Psicología. Universidad de Concepción.

Barnet, B., Joffe, A., Dugan, A., Wilson, M. \& REPKE, J. (1996). Depressive symptoms, stress, and social support in pregnant and postpartum adolescents. Archives of Pediatrics Adolescents Medical, 150, 64-69.

Blumenthal, J., Burg, M., Barefoot, J., Williams, R., Haney, T. \& Zimet, G. (1987) Social Support, Type A Behavior, and Coronary Artery Disease. Psychosomatic Medicine, 49, 331-340.

Brandell, L. \& Cifuentes, M. (2000) Diagnóstico de embarazo adolescente en la Región del Bío Bío, sector salud y educación. Documento no publicado. SERNAM: Concepción.

Brage, D., Elek, S. \& Campbell-Grossman, C. (2000). Depression, self-steem, loneliness and social support among adolescent mothers participating in the new parents project. Adolescence, 35, 445-453.

Brinkmann, H., Segure, M. \& Solar, M. (1989) Adaptación, estandarización y elaboración de normas para el inventario de autoestima de Coopersmith. Revista Chilena de Psicología, 1 (10), 63-68.

Brinkmann, H. \& GARCÈs, M. (1999) Adaptación para adultos del inventario de autoestima de Coopersmith. Ponencia VI Congreso Nacional de Psicología, Santiago, Chile.

Burrows, R., Rosales, M., Ayalo, M. \& Muzzo, S. (1994) Variables psicosociales y familiares asociadas con el embarazo de adolescentes. Revista Médica de Chile, 122, 510-516.

Burrows, R., Rosales, M., Díaz, M. \& Muzzo, S. (1994) Riesgo de embarazo temprano: construcción y validación de un instrumento predictor. Revista Médica de Chile, 122, 713-720. 
Carver, V., Kittleson, M. \& Lacey, E. (1990). Adolescent pregnancy: a reason to examine gender differences in sexual knowledge, attitudes and behavior. Health Values. 14 (6), 24-29.

Castro, M. \& Matamala, C. (1996) Adolescente Embarazada: Apoyo Social Percibido. Un Estudio Sociológico en la comuna de Concepción. Memoria para optar al grado de licenciado en Sociología, Universidad de Concepción.

Coard, S., Nitz, K. \& Felice, M. (2000). Repeat pregnancy among urban adolescents: Sociodemographic, family and health factors. Adolescence, 35, 193-200.

Corcoran, J. (1999) Psychological factors associated with adolescent pregnancy: A review of the literature. Adolescence, 34, 603-619.

Corcoran, J., Franklin, C. \& Bennett, P. (2000). Ecological factors associated with adolescent pregnancy and parenting. Social Work Research, 24 (1), 29-39.

Corona, S. \& Tidwell, R. (1999) Differences between adolescent mothers and nonmothers: An interview study. Adolescence, 34, 91-105.

Cumsille, P. \& Ramírez, V . (1999). Evaluación de un programa comunitario destinado a favorecer el desarrollo psicosocial de madres adolescentes y sus hijos. Psykhe, Revista de Psicología de la Facultad de Ciencias Sociales de la Pontificia Universidad Católica de Chile, 8 (2), 17-29.

D’ Appollonio, J., Díaz, A., Fuenzalida, A., Maldonado, I. \& Mella, R. (2000). Factores asociados a la salud mental en los adultos mayores inscritos en el sistema de salud municipal de la comuna de Talcahuano. Tesis para optar al título profesional de licenciado en Psicología, Universidad San Sebastián.
Del Barrio, V., Moreno-Rosset, C \& LópezMartinez, R. (1999) El Children's Depresión Inventory, (CDI;Kovacs, 1992). Su aplicación en población española. Revista Clínica y Salud, 19, 393-416.

DESMOND, A. (1994).Adolescent pregnancy in the United States: not a minority issue. Health Care for Women international. 15, 325-331.

ERIKSON, E. (2000) El ciclo vital completado. Barcelona: Paidós Ibérica.

Evans, M. \& Rosen, L. (2000). Demographic and psychosocial risk factors for preterm delivery in an active duty pregnant adolescent population. Journal of Medicine. 165, 49-53.

Ferrada M., Molina M. \& Pérez R., Cid, L., Jara, P., Contreras, I., Espinoza, I., LóPez, C. \& Peñalosa, R. (2001). Perfil obstétrico y perinatal en embarazadas adolescentes precoces. VII Congreso Argentino de Perinatología. VI Congreso Latinoamericano de Perinatología, Buenos Aires.

Ferrada M., Molina M.\& Pérez R. (2002). Relación entre la deserción escolar y el embarazo en la adolescencia. Tesis para optar al grado de magíster en ciencias de la educación con mención en administración y gestión educacional. Universidad Mayor, Santiago, Chile.

Florenzano, R. (1994). Familia y salud de los jóvenes. Santiago, Chile: Ediciones Universidad Católica de Chile.

FLORENZANO, R. (1997). El adolescente y sus conductas de riesgo. Santiago, Chile: Ediciones Universidad Católica de Chile.

Fujimori, E., Vianna de Oliveira, I., Rocha, A., Núñez, L. \& Cornbluth, S. (1997) Perfil socioeconómico y biológico de embarazadas adolescentes de una maternidad de beneficiencia en Sao Paulo, Brasil. Cuadernos Médico Sociales. 38, 97-104. 
Gottsegen, E. \& Philliber, W. (2001). Impact of a sexual responsibility program on young males. Adolescence, 36, 427-433.

Gutjarro, S., Naranjo, J., Padilla, M., Gutiérez, R., LAMMERS, C., BLUM, R. W. (1999) Familiy risk factors associated with adolescent pregnancy : study of a group of adolescent girls and their families in Ecuador. Journal of Adolescent Health, 25, 166-172.

Gutierrez, T., Pascacio, E., De la Cruz, A. \& Carrasco, V. (2002). Situación sociofamiliar y nivel de autoestima de la madre adolescente. Revista de Enfermería. 10 (1), 21-25.

Hernández, G., Kimelman, M. \& Montino, O. (2000). Salud mental perinatal en la asistencia hospitalaria del parto y puerperio. Revista Médica de Chile, 128, 1283-1289.

Hoffman, L, PARIS, S. \& Hall, E. (1996). Psicología del desarrollo hoy (6a ed. Vol. 2). Madrid: McGraw-Hill.

Instituto Nacional de Estadísticas. (2000) Enfoques Estadísticos. Boletín informativo del Instituto de Estadísticas de Chile. No 9: Fecundidad. Santiago, Chile.

Instituto Nacional de Estadísticas. (1998) Anuario de Demografía: 1998. Santiago, Chile.

Issler, J. (2001) Embarazo en la Adolescencia. Revista de Postgrado de la Cátedra VI Medicina, 107, 11-23. Disponible en http:// med.edu.ar/revista/revista $107 /$ emb_adolescencia.html.

JaskieWicz, J. \& McAnarney (1994) Pregnancy during Adolescence. Pediatrics in Review, 15 (1). 32-38.

KaLIL, A. \& KunZ, J. (1999) First births among unmarried adolescent girls: Risk and protective factors. Social Work Research, 23 (3), 197-208.
LAgANA, L. (1999) Psychosocial correlates of contraceptive practices during late adolescence. Adolescence, 34, 463-482.

Lartigue, T., Vives, J., Arnaldo, L., LópezDuplán, A., Wohlmuth, R. \& CAsanueva, E. (1992). Características de personalidad de un grupo de adolescentes embarazadas de la ciudad de México. Revista Departamento Psicología. Universidad Iberoamericana, 5 (3-4), 64-76.

Levine, R. \& Chase-Lansdale, P. L. (1998). Adolescent pregnancy and parenthood. American Psychologist, 53, 152-166.

Lipovsek, V., Mehryar, A., Zielinski, E., Magnani, R. \& Castro, M. (2002) Correlates of adoelscent pregnancy in La Paz, Bolivia: Findings from a quantitative-qualitative study. Adolescence, 37, 335-352.

LORBERFELD, Y. (1992) Algunas consideraciones sobre el embarazo adolescente en México. Revista Departamento Psicología Universidad Iberoamericana, 5 (3-4), 100-114.

ManzI, J \& Rosas, R. (1985) Estandarización universitaria del Test de Habilidades Mentales Primarias de T. G. Thurstone. Pontificia Universidad Católica de Chile.

Medora, N. \& Von der Hellen, C. (1997). Romanticim and self-esteem among teen mothers. Adolescence, 32, 811-824.

Miller, C., Miceli, P., Whitman, T. \& BorkowsKI, J. (1996). Cognitive Radiness to parent and intellectual-emotional development in children of adolescent mothers. Developmental Psychology, 32, 533-541.

Millán, T., Yevenez, R., Galvez, M. \& BAHAMONDE, M. (1990). Encuesta sobre síntomas de depresión en embarazadas de un consultorio urbano de atención primaria. Revista Médica de Chile, 118, 1230-1234. 
Molina, M., Peña, I. \& Quiroz, M. (1992) Embarazo precoz. Revista de Trabajo Social. $59,21-28$.

Molina, M., Pérez, R., Ferrada, C., Cid, L., Aguayo, K., Aguilar, F., Astorga, P \& JAQUE, L. (2001). Uso de métodos anticonceptivos en adolescentes embarazadas menores de 15 años. VII Congreso Argentino de Perinatología. VI Congreso Latinoamericano de Perinatología, Buenos Aires.

Molina, M., Salinas, M., Fernández, G., Perez, R. \& Ferrada, C. (1997) El embarazo en la adolescencia. Revista de Ciencia y Salud, 1, 25-29.

Montenegro, H. \& Guajardo, H. (1994). Psiquiatría del niño y del adolescente. Santiago: Salvador.

MuÑoz, M. (1993). Prevalencia, causas y peligros del embarazo entre adolescentes: estrategias preventivas. Revista Niños, 28, 53-64.

Muñoz, B., Berger, C. \& Aracena, M. (2001). Una perspectiva integradora de embarazo adolescente: La visita domiciliaria como estrategia de intervención. Revista de Psicología de la Universidad de Chile, 10, 21-34.

Muzzo, S. \& Burrows, R. (1987) El adolescente chileno. Editorial Universitaria, Santiago.

Out, J. \& Lafreniere, K. (2001) Baby Think It Over ${ }^{\circledR}$ : Using Role-Play to prevent teen pregnancy. Adolescence, 36, 571-582.

Papalia, D. \& Wendoos, S. (1987) Psicología. Editorial McGraw Hill, Bogotá, Colombia.

Papalia, D. \& WendKos, S. (1998). Psicología del desarrollo (7a. ed.). Bogotá: McGraw Hill.

Pinto, L., Gallardo, I. \& Wenk, E. (1991) Determinación denormas para el PMA de L.L. Thurstone en estudiantes de cuarto año de enseñanza media científico humanista de la Región metropolitana, Revista de Psicología de la Universidad de Chile, 1(2), 25-42.
Porras, A. (1994). Condiciones del embarazo de adolescentes primigestas en Siquirres, Limon. Revista de Ciencias Sociales, (65), 57-66.

Pozo, J. (1997). Teorías cognitivas del aprendizaje. Madrid: Morata.

Ramírez, V. \& Cumsille, P. (1997). Evaluación de la eficiencia de un programa comunitario de apoyo a la maternidad adolescente. Revista Latinoamericana de psicología, 29(2). 267- 286.

RAVERT, A. \& MARTíN, J. (1997) Family stress, perception of pregnancy, and age of first menarche among pregnant. Adolescence, $32,261-269$.

RESTREPO, M. (1991) Factores psicológicos en madres adolescentes: intervención dentro de un programa de atención primaria en salud. Revista de Psicología, 26, 57-71.

RICE, F. (1997). Desarrollo Humano. (2a ed.) México. Editorial Prentice-Hall Hispanoamérica.

RojAs, A. (1991) Problemática psicosocial de la madre adolescente. Revista Ciencias Sociales, 53, 75-82.

Salinas, E. \& Armengol, G. (1992) El autoconcepto en la adolescente embarazada. Revista Departamento Psicología, Universidad Iberoamericana, 5 (3-4), 90-99.

Scaramella, L., Conger, R., Simons, R. \& Wнітвеск, L. ( 1998). Predicting risk for pregnancy by late adolescence: a social contextual perspective. Develomental Psychology, 34 (6). 1233- 1245.

Short J., \& Slusher I. (1994). Adolescent pregnancy. Kentucky Nurse. 42 (3). 36-37.

Steven-Simon, C. \& Lowy, R. (1995) Teenage childbearing. An adaptative strategy for the socioeconomically disadvantaged or a strategy for adapting to socioeconomic disadvantage?. Archives of Pediatrics Adolescents Medical, 149, 912-915. 
TRAD, P. (1999) Assesing the patterns that prevent teenage pregnancy. Adolescence, 34, 221-240.

Troutman, B. \& Cutrona, C. (1990). Nonpsychotic postpartum depresion among adolescent mothers. Journal of Abnormal Psychology, 99, 69-78.

Universidad de Chile, Centro de Políticas PúBlicAs. (2000). Resumen. Tercer informe avance propuesta de política pública para la prevención del embarazo en adolescentes y apoyo al desarrollo de su sexualidad y afectividad. Preparado para Servicio Nacional de la Mujer. Comisión Interministerial para la Prevención del Embarazo Adolescente. Santiago: Autor.

Varas, J., Kramarosky, C., Díaz, A., Sibilla, M. \& GAETE, R. (1999) Embarazo en adolescentes: aspectos bio-psico-socio-sexuales. Revista Chilena de Obstetricia y Ginecología. 64, 438-443
Venegas, M., Hidalgo, M., Hurtado, M. \& Nova, C. (2001)Factores familiares e individuales predisponentes de la anorexia nerviosa en adolescentes mujeres alumnas de enseñanza media de colegios particulares, particulares-subvencionados y liceos municipalizados de la Comuna de Concepción, en el año 2001. Tesis para optar al grado de Licenciado en Psicología, Universidad San Sebastián, Concepción, Chile.

Vera, G., Gallegos, M. \& Varela, M. (1999). Salud Mental y Embarazo en adolescentes. Contexto de la gestación y consecuencias biográficas tempranas. Revista Médica de Chile, 127, 437-433.

Vives, J., Lartigue, T., Díaz-Barriga, M., Legarreta, D., Salinas, E. \& Casanueva, E. (1992) Factores precipitantes del embarazo en adolescentes. Revista Departamento Psicología, Universidad Iberoamericana, 5(3-4), 77-89. 